\title{
A Review on the Development of Rotman Lens Antenna
}

\author{
Shruti Vashist, ${ }^{1}$ M. K. Soni, ${ }^{2}$ and P. K. Singhal ${ }^{3}$ \\ ${ }^{1}$ FET, ECE-Department, Manav Rachna International University, Faridabad, Haryana 121001, India \\ ${ }^{2}$ Manav Rachna International University, Faridabad, Haryana 121001, India \\ ${ }^{3}$ HOD-ECE, MITS, Gwalior, India \\ Correspondence should be addressed to Shruti Vashist; shruti.fet@mriu.edu.in
}

Received 17 February 2014; Revised 19 June 2014; Accepted 20 June 2014; Published 17 July 2014

Academic Editor: Dean Deng

Copyright (C) 2014 Shruti Vashist et al. This is an open access article distributed under the Creative Commons Attribution License, which permits unrestricted use, distribution, and reproduction in any medium, provided the original work is properly cited.

\begin{abstract}
Rotman lenses are the beguiling devices used by the beamforming networks (BFNs). These lenses are generally used in the radar surveillance systems to see targets in multiple directions due to its multibeam capability without physically moving the antenna system. Now a days these lenses are being integrated into many radars and electronic warfare systems around the world. The antenna should be capable of producing multiple beams which can be steered without changing the orientation of the antenna. Microwave lenses are the one who support low-phase error, wideband, and wide-angle scanning. They are the true time delay (TTD) devices producing frequency independent beam steering. The emerging printed lenses in recent years have facilitated the advancement of designing high performance but low-profile, light-weight, and small-size and networks (BFNs). This paper will review and analyze various design concepts used over the years to improve the scanning capability of the lens developed by various researchers.
\end{abstract}

\section{Introduction}

To appreciate the significance of the most popular constrained lens architecture, the Rotman lens antenna, it is necessary to provide a review of the constrained lens development. To fully understand the concept of these complicated and complex lenses, the basic concept of microwave beamforming network must be dealt with in detail. The Rotman lens antenna provides ideal performance for applications such as for satellite based direct radiating arrays, remotepiloted vehicles, collision-avoidance radars, ultra-wideband communications systems, and many more $[1,2]$. Constrained lens arrays are special groups of beamforming networks. They share some similarities with dielectric lenses and reflector antennas on one hand and with antenna arrays on the other. Their function is to form beams in multiple directions which correspond to the position of the feed antennas at the focal surface. The name constrained comes from the fact that a wave incident on one face of the array does not necessarily obey Snell's law when passing through the lens array [1]. It is instead constrained to follow the transmission line paths. Unlike dielectric lenses or reflector antennas, lens arrays do their collimation (transmission) and focusing (reception) discretely, using antenna arrays [2].
The frequency independent beam pointing angle characteristics of the lens due to its time delay nature makes it ideal for many broadband width electronics warfare.

The paper is organized as follows.

Section 2 represents various beamforming techniques and its development. Section 3 deals with the design equations of the lens. Section 4 presents the various design approaches of the Rotman lens and finally Section 5 is the conclusion.

\section{Development of the Beamforming Networks}

The device that produces dynamic feeding information for an aperture is called a beamforming network (BFN). The beamforming network usually consists of $M$ number of input ports and $N$ number of output ports that are subjected to feed $N$ number of array elements [4]. Typical layout is shown in Figure 1. A BFN produces desirable amplitude and phase distributions across the aperture for each input port so that quality beams can be sent into different angles. A beamforming network can be planar or 3-dimensional, depending on the aperture requirement. Usually, 2D BFN produces $2 \mathrm{D}$ steerable fan beams, while $3 \mathrm{D}$ BFN generates $3 \mathrm{D}$ steerable pencil beams. 
TABle 1: Different types of beamforming networks.

\begin{tabular}{|c|c|c|c|c|}
\hline S. number & BFN & Features & Advantages & Disadvantages \\
\hline 1 & $\begin{array}{l}\text { Digital } \\
\text { beamforming } \\
\text { networks }\end{array}$ & $\begin{array}{l}\text { They make use of a computer or } \\
\text { chip processor to control } \\
\text { electronic components forming } \\
\text { exact amplitude and phase for } \\
\text { different channels of array } \\
\text { elements. }\end{array}$ & $\begin{array}{l}\text { They have zero phase error, } \\
\text { flexible amplitude tapering, and } \\
\text { infinite number of scanning } \\
\text { steps. }\end{array}$ & $\begin{array}{l}\text { They are limited to } \\
\text { low-microwave frequencies due } \\
\text { to low bit-bandwidth of current } \\
\text { A/D devices [8]. It requires fast } \\
\text { processors in order to form a } \\
\text { coherence receiver. In case where } \\
\text { large processing of information is } \\
\text { involved, the computation speed } \\
\text { becomes difficult. }\end{array}$ \\
\hline 2 & $\begin{array}{l}\text { Network } \\
\text { beamforming } \\
\text { network }\end{array}$ & $\begin{array}{l}\text { One of the earliest BFNs which } \\
\text { originated from the Butler } \\
\text { matrix. It consists of alternate } \\
\text { rows of fixed phase shifters and } \\
\text { hybrid junctions }[8,9] \text {. }\end{array}$ & $\begin{array}{l}\text { They are easy to construct and } \\
\text { can be implemented using } \\
\text { printed circuit boards. The } \\
\text { produced beams are dependent } \\
\text { on frequency. }\end{array}$ & $\begin{array}{l}\text { It requires crossovers on the } \\
\text { lines. The beam shift occurs as } \\
\text { the frequency varies, which is not } \\
\text { desirable in most } \\
\text { communications links, as certain } \\
\text { bandwidth information is } \\
\text { expected to be sent to the same } \\
\text { location. }\end{array}$ \\
\hline 3 & $\begin{array}{l}\text { Microwave lens } \\
\text { beamforming } \\
\text { network }\end{array}$ & $\begin{array}{l}\text { It applies path delay mechanism } \\
\text { to form the desired phase front at } \\
\text { the array input. Each of the input } \\
\text { connects to a beam port that } \\
\text { radiates semicircular phase front } \\
\text { within the lens cavity [10]. Array } \\
\text { of receiving elements functioning } \\
\text { as receivers then guides the } \\
\text { energy into the output array. }\end{array}$ & $\begin{array}{l}\text { The path-length design } \\
\text { mechanism in the microwave } \\
\text { lens is independent of frequency } \\
\text { [10]; thus it is typically } \\
\text { considered as true-time delay } \\
\text { device [11]. The properly } \\
\text { designed beam, receiving port } \\
\text { positions, transmission line } \\
\text { length, correct phase, and } \\
\text { acceptable amplitude } \\
\text { distributions can be achieved } \\
\text { across the aperture. }\end{array}$ & $\begin{array}{l}\text { Finite insertion losses and } \\
\text { inherent losses occur due to the } \\
\text { hybrid couplers, fixed phase } \\
\text { shifters, and transmission lines } \\
\text { that make up the matrix. The } \\
\text { Butler matrix passive beam } \\
\text { forming antenna also requires } \\
\text { that the individual beam patterns } \\
\text { be orthogonal in space. }\end{array}$ \\
\hline
\end{tabular}

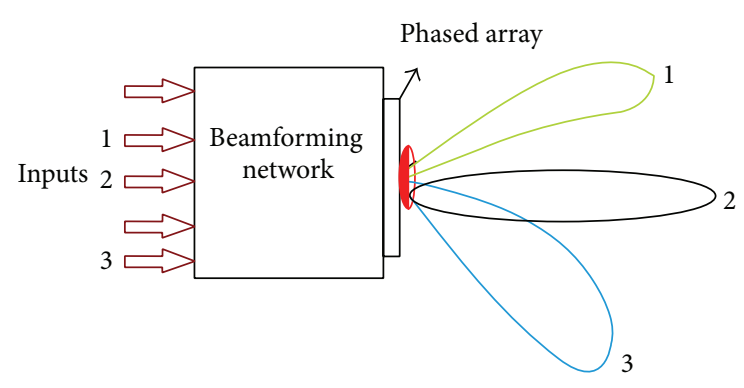

FiguRE 1: Beamforming network.

There are several ways of designing and categorizing the BFNs. They are the digital BFNs, network BFNs, and microwave lens BFNs. The Rotman lens antenna design falls in the regime of lens BFNs. Few highlights, advantages, and drawbacks of different BFNs are given in Table 1.

2.1. History of Microwave Lens as Beamforming Network (See Figure 2). Figure 2 shows the typical characteristics of the microwave lens. Assume that an RF signal is being received by an antenna array with $N$ number of elements, from a particular direction. Due to certain geometry of the antenna array, the impinging RF signal reaches the individual antenna elements at different instants of time, which causes phase

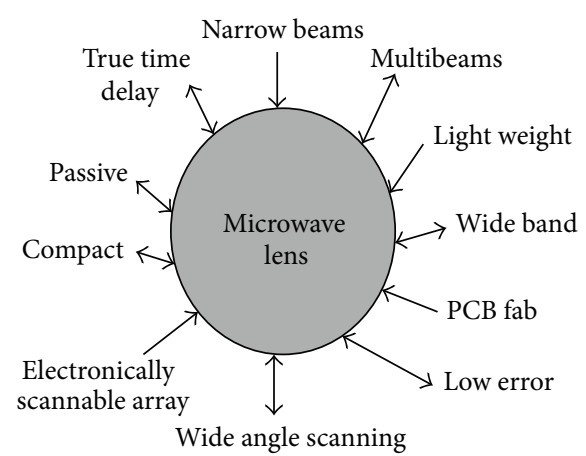

FIGURE 2: Characteristics of microwave lens.

shifts between the different received signals. Using microwave beamforming technique, the beam patterns of the antenna array can be steered in certain desired directions, whereas undesired directions can be suppressed. Microwave lens emerged as a beamforming network (BFN) in the 1950s, and it subsequently was involved in numerous cutting edge applications [5,6]. Advancements in materials science and fabrication technologies led to new implementations of the microwave lenses using waveguide, striplines, and microstrip. Many applications including automobile collision-avoidance, remotely piloted radars [7], and remote satellite sensor require compact, lightweight antenna systems capable of 
providing wide-angle scanning over a broad frequency bandwidth.

The Rotman lens is an attractive passive microwave lensbased beamforming network due to its low cost, reliability, design simplicity, and wide-angle scanning capabilities [12]. It is a device that uses the free-space wavelength of a signal injected into a geometrically configured waveguide to passively shift the phase of inputs into a linear antenna array in order to scan a beam in any desired signal pattern. It has a carefully chosen shape and appropriate length transmission lines in order to produce a wave-front across the output that is phased by the time-delay in the signal transmission. A Rotman lens achieves beam scanning using equivalent timedelays that are created by the different path lengths to the radiating elements. Since Rotman lens is a true-time-delay (TTD) device, it produces beam steering independent of frequency and is therefore capable of wide-band operation. Researches on intelligent transportation systems and applications were carried out to enhance safety and efficiency of road transportation related to vehicle-to-vehicle (V2V) and vehicle-to-infrastructure (V2I) automotive communications. Microwave beamforming networks can greatly increase and enhance the performance of wireless systems used in intelligent transportation systems and framework. In this contribution, passive planar steerable microwave beamforming networks based on Rotman lens and cascaded Butler matrices have been designed and analyzed in intelligent transportation systems [13].

These lengths depend on the relative position between the beam port and the array ports on the structure. As long as the path lengths exhibit constant time-delay behavior over the bandwidth, the lens is insensitive to the beam squint problems exhibited by constant phase beamformers. Each input port will produce a distinct beam that is shifted in angle at the system output.

The design of the lens is controlled by a series of equations that set the focal points and array positions. The inputs, during the design of the system, include the desired number of beams and array elements and the spacing of the elements.

\section{Rotman Lens Design Parameters}

A Rotman lens is built using microstrip techniques, feeding a patch antenna array. It satisfies the qualities required in an antenna as it provides high gain, large scan angles, conformal geometry, and low cost. There is a lot of scope in optimizing various parameters which are useful in designing Rotman lens antenna. The antenna is capable of producing multiple beams which can be optimized to steer without changing the antenna orientation. Figure 1 shows the basic diagram of the Rotman lens [14]. It consists of a set of input and output ports arranged along an arc. It consists of a parallel plate region surrounded by number of array ports and beam ports. Dummy loads can also be introduced to provide reflection less termination of parallel plate region. Dummy ports are also an integral part of the Rotman lens and serve as an absorber for the spillover of the lens and thus it reduces multiple reflections and standing waves which deteriorate

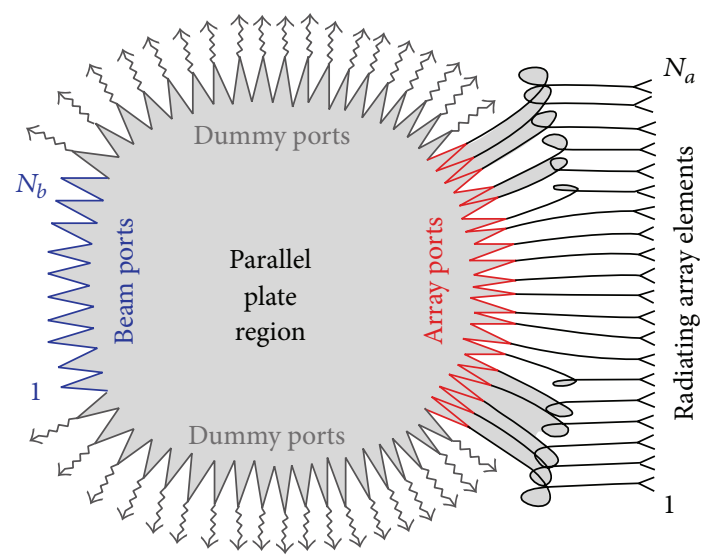

Figure 3: Basic construction of Rotman lens.

the lens performance. The lens structure between both sets of ports functions as an ideal transmission line between the individual input and output ports. The signal applied to the input port is picked up by the output port.

The different electrical lengths between a specific input and all output ports generate a linear progressive phase shifts across the output ports of the lens. The design of the lens is governed by the Rotman-Turner design equations that are based on the geometry of the lens as shown in Figure 3.

Figure 4 shows a schematic diagram of a trifocal Rotman lens. Input ports lie on contour $C_{1}$ and the output ports lie on contour $C_{2} . C_{1}$ is known as beam contour and $C_{2}$ is known as array contour. There are three focal points, namely, $F_{1}, F_{2}$, and $G$. $G$ is located on the central axis while $F_{1}$ and $F_{2}$ are symmetrically located on the array contour at an angle of $+\alpha$ and $-\alpha$, respectively. It is quite clear from Figure 2 that the coordinates of two off-axis focal points $F_{1}$ and $F_{2}$ and one onaxis focal point $G$ are $(-F \cos \alpha, F \sin \alpha),(-F \cos \alpha,-F \sin \alpha)$, and $(-G, 0)$, respectively [15]. The equations generate the positions of the antenna ports based on three perfect focal points $\left(G, F_{1}\right.$, and $\left.F_{2}\right)$. The following equations govern the design of the lens:

$$
\begin{gathered}
F_{1} P \sqrt{\varepsilon_{r}}+W \sqrt{\varepsilon_{e}}+N \sqrt{\varepsilon_{i}} \sin \alpha=F \sqrt{\varepsilon_{r}}+W_{0} \sqrt{\varepsilon_{e}}, \\
F_{2} P \sqrt{\varepsilon_{r}}+W \sqrt{\varepsilon_{e}}-N \sqrt{\varepsilon_{i}} \sin \alpha=F \sqrt{\varepsilon_{r}}+W_{0} \sqrt{\varepsilon_{e}}, \\
G P \sqrt{\varepsilon_{r}}+W \sqrt{\varepsilon_{e}}=G \sqrt{\varepsilon_{r}}+W_{0} \sqrt{\varepsilon_{e} .}
\end{gathered}
$$

The design parameters for the lens with different substrates in the cavity, transmission line and the inner receiving contour are represented in Table 2 and with the air filled in the cavity is represented in Table 3.

The above equations provided the method to design the simple first order design for Rotman lens. The original Rotman lens was developed in 1963 [15]. It achieved twodimensional scanning using a parallel plate transmission medium for true time delay architecture. In the paper Rotman suggested that his design of the lens would show better performance in terms of scanning angle in fractional degree steps out performing the state of art at that time. His work improved on coma aberrations and off-axis phase distortions 


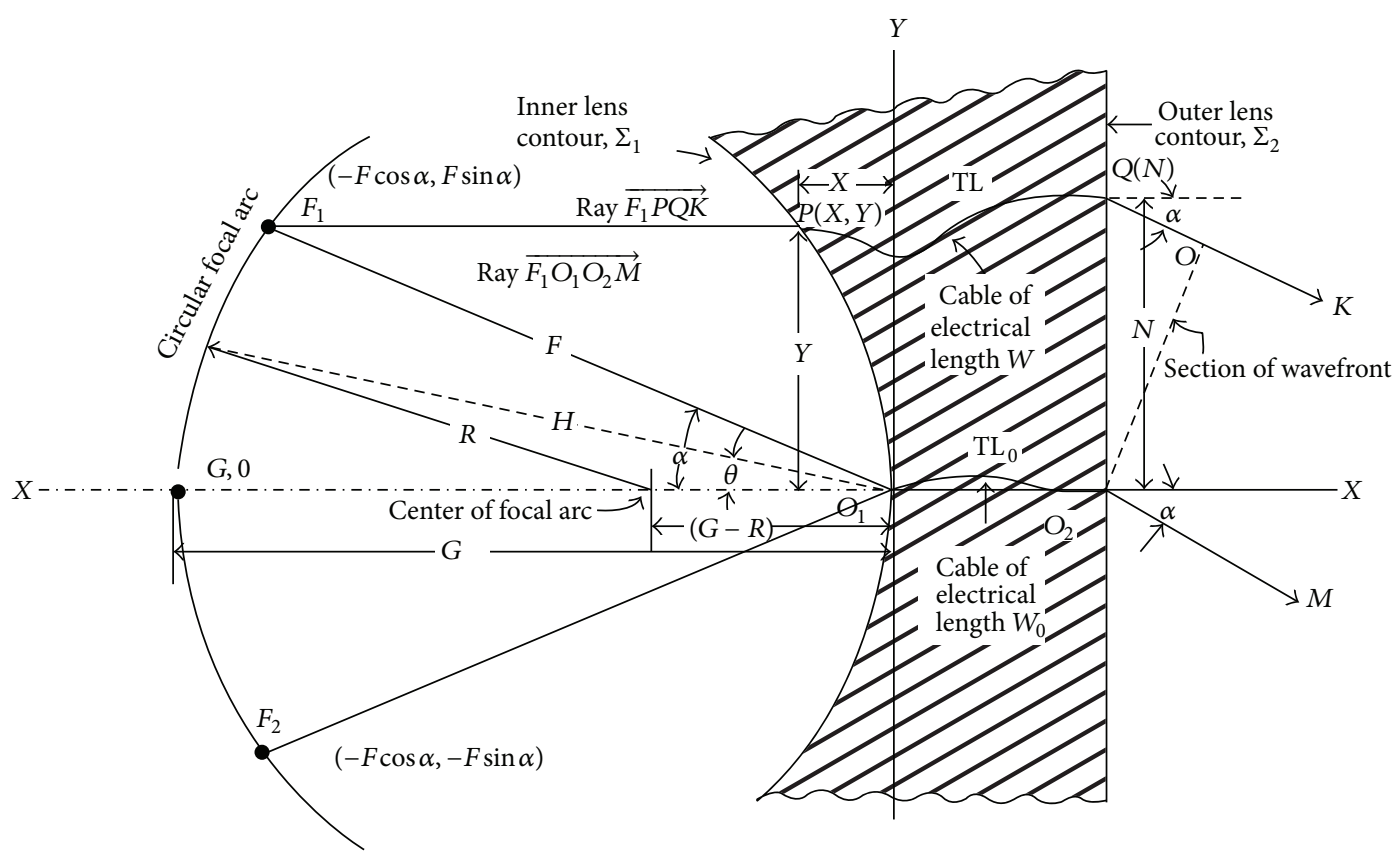

Figure 4: Geometry and design parameters of Rotman lens.

TABLE 2: Design parameters for the lens with substrate.

$x=\frac{w \sqrt{\varepsilon_{e}}(1-g)}{\left(g-a_{o}\right) \sqrt{\varepsilon_{r}}}-\frac{n^{2} \varepsilon_{i} b_{o}^{2}}{2\left(g-a_{o}\right) \varepsilon_{r}}$
$y=\frac{n \sqrt{\varepsilon_{i}}}{\sqrt{\varepsilon_{r}}}\left(1-\frac{w \sqrt{\varepsilon_{e}}}{\sqrt{\varepsilon_{r}}}\right)$
$a=\frac{\varepsilon_{e}}{\varepsilon_{r}}\left(\frac{(1-g)^{2}}{\left(g-a_{o}\right)^{2}}+\frac{n^{2} \varepsilon_{i}}{\varepsilon_{r}}-1\right)$
$b=\frac{\sqrt{\varepsilon_{e}}}{\sqrt{\varepsilon_{r}}}\left[\left(\frac{(1-g)}{\left(g-a_{o}\right)} * \frac{n^{2} \varepsilon_{i} b_{o}^{2}}{\left(g-a_{o}\right) \varepsilon_{r}}\right)-\frac{2 n^{2} \varepsilon_{i}}{\varepsilon_{r}}+2 g+\frac{2 g(1-g)}{\left(g-a_{o}\right)}\right]$
$c=\frac{n^{4} \varepsilon_{i}^{2} b_{o}^{4}}{4\left(g-a_{o}\right)^{2} \varepsilon_{r}^{2}}+\left(\frac{n^{2} \varepsilon_{i}}{\varepsilon_{r}}\right)-\frac{g n^{2} \varepsilon_{i} b_{o}^{2}}{\left(g-a_{o}\right) \varepsilon_{r}}$
$w=\frac{-b \pm \sqrt{b^{2}-4 a c}}{2 a}$

of the general lens principles that were developed by Jones et al. by using there perfect focal points at the input side [3].

The history of microwave lens design familiarizes us with how previous researchers improved the design of microwave lenses and their visions upon the development. Furthermore, reviewing the past and emerging applications of microwave lens helps form and assess the usefulness of the newly proposed designs. Gent obtained generalized design equations for arbitrary lens shapes and by using lens equations [3] Rotman and Turner introduced Rotman lens phenomena.

In the following section of the paper, historical developments, work done, and various applications of Rotman lens will be discussed in detail. The detailed contributions of work done by other researchers helped a lot to develop the review paper.
TABLE 3: Design parameters with air filled in the cavity.

$$
\begin{aligned}
& x=\frac{w(1-g)}{\left(g-a_{o}\right)}-\frac{n^{2} \varepsilon_{i} b_{o}{ }^{2}}{2\left(g-a_{o}\right)} \\
& y=n(1-w) \\
& a=\left(\frac{(1-g)^{2}}{\left(g-a_{o}\right)^{2}}+n^{2}-1\right) \\
& b=\left[\left(\frac{(1-g)}{\left(g-a_{o}\right)} * \frac{n^{2} b_{o}^{2}}{\left(g-a_{o}\right)}\right)-2 n^{2}+2 g+\frac{2 g(1-g)}{\left(g-a_{o}\right)}\right] \\
& c=\frac{n^{4} b_{o}^{4}}{4\left(g-a_{o}\right)^{2}}+n^{2}-\frac{g n^{2} b_{o}^{2}}{\left(g-a_{o}\right)} \\
& w=\frac{-b \pm \sqrt{b^{2}-4 a c}}{2 a}
\end{aligned}
$$

\section{Various Design Approaches of Rotman Lens Antenna}

Microwave lens is a structure which is capable of focusing the electromagnetic energy into a point. The microwave region defines the region with the electromagnetic spectrum with wavelength between $1 \mathrm{~mm}$ and $300 \mathrm{~mm}$. During its development in 1950s, metallic plate and constrained line techniques were originally adopted in designing the Ruze lens [16] and the R-2R microwave lens. In 1957, a general theory of improved constrained lens was invented by Gent [15], and it was named bootlace lens because of its general structure as shown in Figure 5. Rotman lens was introduced by Rotman and Turner in 1960s. The lens was designed with mainly three focal points and hence there was an improvement in the phase error performance. After the invention of Rotman lens, in Raytheon Electronic Warfare division, systems based on 


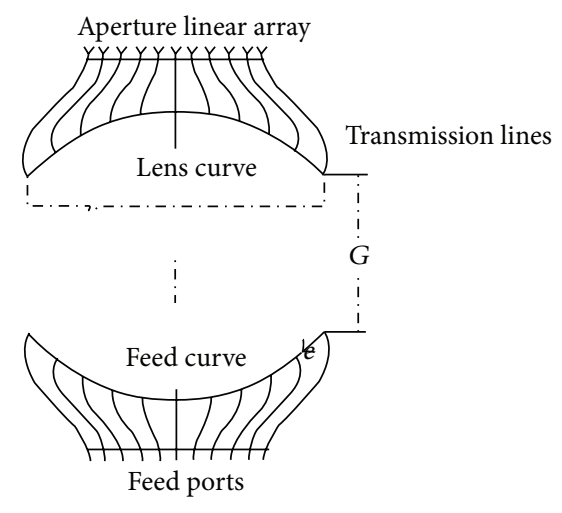

FIgURE 5: Gent bootlace lens configuration.

Rotman lens in 1967, the work was done on reducing the size of the lens by loading the parallel plate region by dielectric material [17]. In 1967 first microstrip Rotman lens and scan expansion was introduced. It was observed that if the parallel plates were loaded uniformly with the dielectric material with typical permittivity $\varepsilon_{r}$, then the linear dimensions of the lens would come down by a factor $1 / \varepsilon_{r}[18]$. It provided additional degree of freedom to the designer. This factor was called an expansion or compression factor.

In 1970, first 2D Rotman lens stack was demonstrated, due to the independence of the mutually orthogonal array factors comprising a planar array rectangular lattice, it was possible to arrange Rotman lens in 2D stacks [19]. In 1971 MUSTRAC, a shipboard telemetry tracking system was developed.

In 1960, Rotman and Tuner applied Gent's general lens design schemes in their early warning radar systems [3, 15]; in 1962 they reported a three perfect focal point microwave lenses, which had greatly improved the phase error and design freedoms of the original Ruze lens [16] as shown in Figure 3.

Keeping in view the model's fundamental limits, researchers have developed and modified three focal lenses [15], four focal lenses [20], and nonfocal lenses [21]. The conventional models and their development are briefly addressed in the following sections.

4.1. The Rotman Lens Design Approach by Rotman and Turner. The review begins with the survey of the conventional Rotman lens design (RLD) [15]. The original RLD consists of a parallel conducting plate, fed by horn antennas along the beam contour, and transmission lines that connects the inner receiver contour and the outer phased arrays, as shown in Figures 6 and 7. The lens was designed for feeding linear arrays. There were three focal points located on the beam contour, which generated zero theoretical phase errors along the phase front of the linear array elements. In the original model the parallel plate was assumed air filled. The beam contour was restricted to be circular and the scan angle produced by linear array was constrained to be of the same value as the subtended beam port angle.

Research work on Rotman lens antenna started way back in 1963 when Rotman and Turner published their research

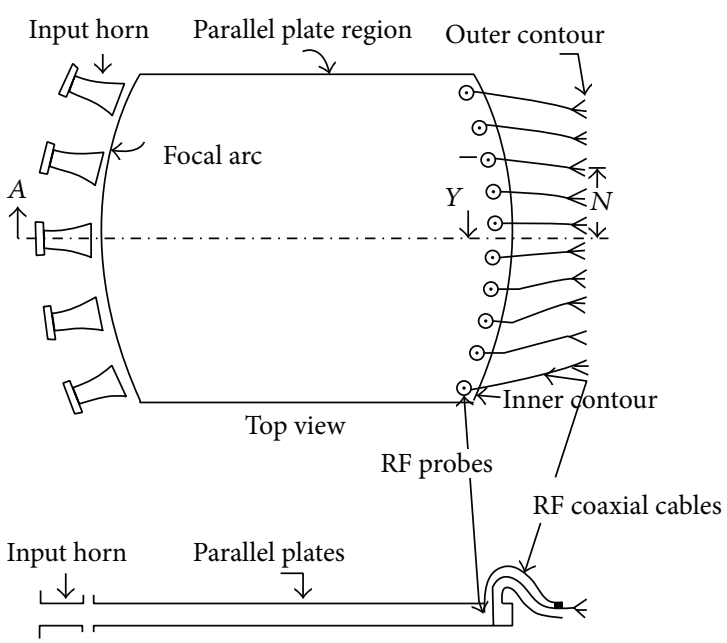

FIgURE 6: Original Rotman lens configuration.

work. This work still remains the bench mark for researchers in this area.

The lens parameters are defined as shown in Figure 7. The focal arc locates the feeding elements which are termed as the beam port or the beam contour. The inner lens contour locates the receiving elements and the outer lens contour or the array port locates the radiating array elements. For the beam contour design, three focal points were used: two off-axis focal points $\left(F_{1} \& F_{2}\right)$ which were symmetrical and one on-axis focal point $(G)[15]$. The shape of the focal arc was chosen as a circular. Unlike the other types of lenses, including the Ruze model for which the parameters $Y$ (the $y$-coordinate of an arbitrary point on the inner lens contour) and $N$ (the coordinate of a radiating array element connected to the receiving element locating at $P(X, Y)$ ) were kept equal to each other; Rotman lens allowed $Y$ and $N$ to be different. This provided more degrees of freedom in the design. In order to derive design equations for the lens contour, optical pathlength equality and the lens geometry were used.

4.2. Symmetrical Lens Approach by Shelton (See Figure 8). Shelton [22] developed a symmetrical lens configuration as a modification to the Rotman lens. The beam and the inner lens contours were identical and symmetrical with respect to a symmetry plane as shown in Figure 8. This design was useful for comparable number of input and output ports. The design equations of this type of lens were more complicated than that of Rotman.

Between 1981 and 1983 it was experimentally determined by Maybell [23] that for the lens port width between 0.5 and 0.7 the active reflection coefficient of the port increases significantly at small angles as 15 degrees and results in severe degradation of the lens amplitude and phase distributions.

The solution to the above problem was given in 1984 in the form of patent by Hardie et al. [24]. They suggested that lens port suffering with this problem is to be replaced by half width port pair and an isolated power combiner.

In 1984 Katagi et al. [25] improvised the design equations given by Rotman and Turner by adding a new variable which 


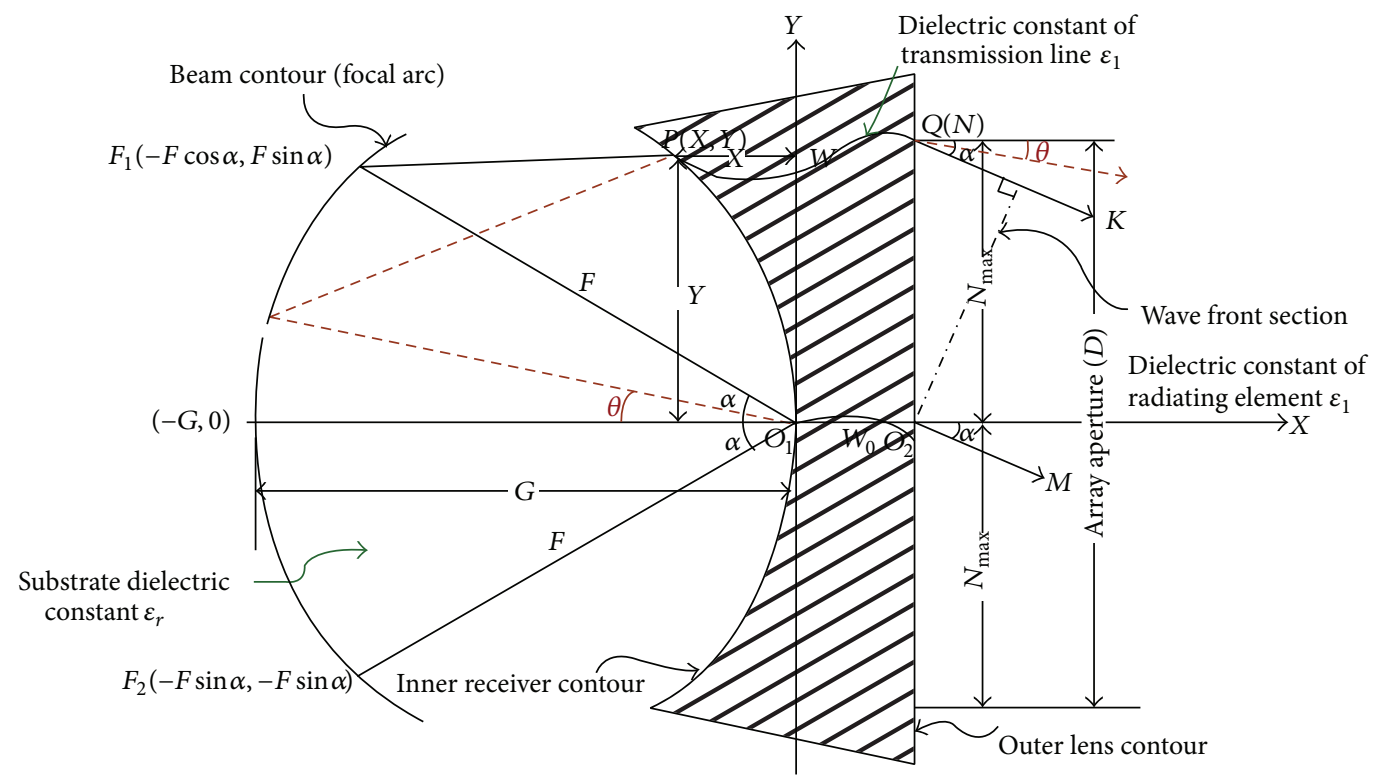

Figure 7: Rotman lens geometry.

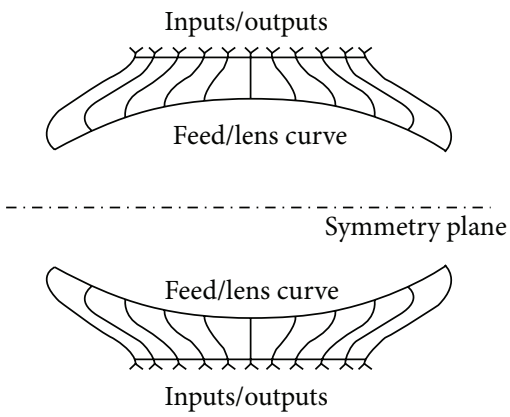

FIgURE 8: Symmetrical lens.

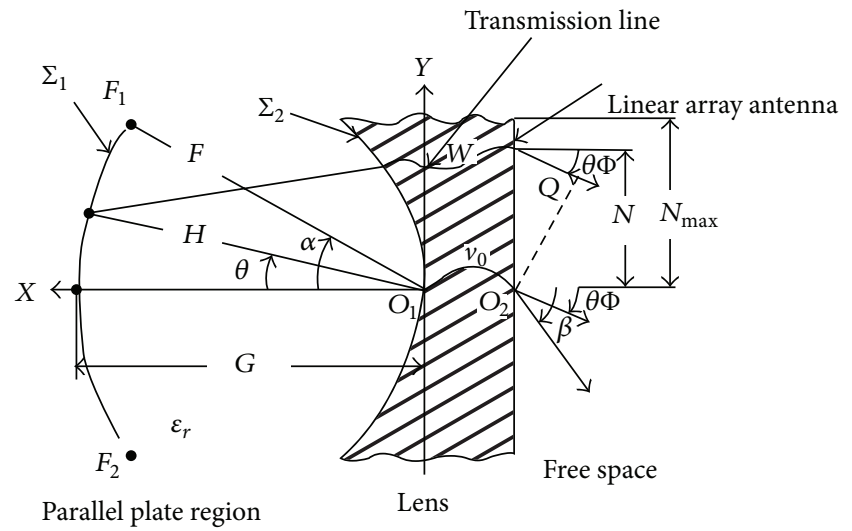

FIGURE 9: Katagi's design of the lens.

reduced the phase error on the aperture of the linear array antenna. This design parameter helped in reducing the size of the lens.

As it could be seen in Figure 9, Katagi defined a subtended angle $(\alpha)$ corresponding to one of the off-axis focal points as was defined in Rotman's model. However, the scan angle $(\beta)$ corresponding to the excitation from $F_{1}$ was assumed to be different from the subtended angle $(\alpha)$ though scan angles were assumed to be equal to the corresponding subtended angles in Rotman's design model. Hence, Katagi introduced a new design variable consisting of the ratio of the scan angle and the subtended angle corresponding to one of the off-axis focal points. This variable provided a new degree of freedom compared to the conventional design. Katagi also suggested that the shape of the beam contour is not necessarily a circular arc. Based on the concept of Rotman lens design, Katagi proposed an improved design in 1983. By applying a refocus method, the modified equations theoretically demonstrated that changing the circular contour and altering the radiation angles yielded lower phase errors than the original design.
In 1989 Musa and Smith presented a new design approach for reduction of side wall absorption which was one of the performances limiting parameter of the lens [26, 27]. Musa and Smith emphasized the flexibility of designing large scan angle Rotman Lens by applying small subtended beam regions. The path-length equation was firstly expressed in a power series and the importance of focal length $R$ factor was studied. In later work, the Snell equations and 1D aperture theory were first time used to predict the amplitude performance of the Rotman lens. A prototype of waveguide lens, designed based on conventional three focal methods, without side wall assumptions, was conducted. Both simulation and measurement demonstrated cosine shape tapered amplitude distributions.

In 1990 Hall et al. reviewed radio frequency beamforming techniques and presented a very wide range of solutions incorporating both quasioptics and circuit base type networks [28]. They found that the reflectors and lenses produce 
high gain beams with very narrow scan ranges. Circuit beam formers have the well-known traveling wave or corporate feed characteristics and could be used in limited size array which in addition gave wide bandwidth.

4.3. Design Trades by Hansen. A big breakthrough in this work came in 1991. Hansen [14] used six basic design parameters: focal angle, focal ratio, beam angle to ray angle ratio, maximum beam angle, focal length, and array element spacing. One more parameter of ellipticity was introduced which assumed the beam contour to be elliptical instead of circular. The parameters beam angle (subtended angle) to ray angle (scan angle) ratio and ellipticity are additions to the parameters of the conventional design. Hansen explained the effects of the seven design parameters on the shape and on the geometric phase and amplitude errors of a Rotman lens in detail. The general design equations and parameters were reformulated in Hansen's work. In this design trade, the elliptical beam contour was introduced, and the relation between the scan angle and the beam subtended angle was captured by the parameter defined as $\gamma=\sin \Psi / \sin \alpha$. Relation between the phase errors and typical design parameters was studied, and the optimization factor $\beta$ was finalized. In Hansen's work, the lens was assumed to be air filled and beams were scanned into free space.

4.4. Refocusing Approach by Gagnon, Modified by Singhal. Gagnon introduced refocusing procedure for dielectric-filled Rotman lens according to Snell's law [29]. Therefore, applying Snell's law yields a ratio of $\sqrt{\epsilon}$ between the sine of the scan angle and the subtended angle of the beam contour. This approach provided beam and array port positions which gave improved coupling to the outermost beam ports, especially for printed lenses used with small arrays. A modified approach to design Rotman (1963) type multiple beamforming lens had been proposed by Singhal et al. [30]. The path length error at the wavefront was calculated. The error obtained by the proposed approach and by the conventional approach has been compared. Two-dimensional electromagnetic field analysis of the lens obtained by the modified approach and by the conventional approach lens was carried out using the contour integral method. The results obtained for both the lenses were compared.

In 1995 Woodward synthesis was given by Maybell [31] that would excite Rotman lens adjacent beam ports with amplitudes adjusted to sample a desired beam shape. Low side lobes and constant widths were obtained over greater than octave bandwidths.

In 1996 Rausch and Peterson proposed a design based on the contour integral. An mm wave Rotman lens that operates between the frequency of 33 and $37 \mathrm{GHz}$ was designed [32]. Various parameters were analyzed. Reduction in side lobe level and insertion loss was observed. Greater scanning angles were possible with different lens design. In the same year, multibeam array using Rotman lens and RF heterodyne was proposed by JJ Lee. RF heterodyne technique was applied to Rotman lens to reduce the size of beamforming network for airborne antenna operating at L-band $(1.4 \mathrm{GHz})$.
In 1996 low cost compact electronically scanned mm wave antenna was proposed by Rausch and Peterson which showed good reduction inside lobe levels and insertion loss [32].

In 2003 Singhal et al. proposed the fact that the height of the array and feed contours must be the same for maximum power transfer and better lens performance [33]. Effect on shape of beam and array contour by variation in scanning angle, focal ratio, and element spacing were prime issues of his work.

Simon in 2004 analyzed the performance of the lens using his own simulation tool designed in MATLAB platform. It was probably the first reported GUI specially designed for Rotman lens antenna [34]. Accuracy of author's proposed Rotman lens design software is verified by comparing its results with numerical analysis of Rotman lens (NARL).

The quadrifocal bootlace lenses were first proposed to design 3-dimensional lens beams forming networks by Rao $[20,21]$. The lens consisted of four perfect focal points along a linear feeding line and a nonplanar output phased array. Dong and Cheung [35] applied this technique to design a circular feeding contour and linear output 2-dimensional lens. Different from the RLD, the planar lens allows 4 symmetric perfect focal points allocated along the beam contour, and this arrangement can still feed the same number of phased array elements at the output. Beam contour perturbation was the technique adopted in [36] to improve the phase performance of the quadrifocal planar lens. It is noted that the lens equations only support designs of air filled lens and the scan angle is also restricted to be the same with the subtended angle.

In 2009 nonfocal lens design and 360-degree scanning lens were done by Dong. He reported a design of a microwave lens which had the capability of 360-degree scanning. This was a major breakthrough since work of this kind was never reported where lens had achieved the capability of complete 360-degree scanning. The conventional Rotman lens phase error could be reduced by adapting a nonfocal design strategy, proposed by Dong [37-39] in 2008. The proposed method allowed designing a Rotman lens phase center contour without passing any ideal foci. The original Rotman lens [15] was designed to have 3 ideal focal points, which led to the design of a deterministic inner receiving contour that gave zero path-length errors for the beams emitted from these 3 focal points. However, in the realistic design, more beam elements were expected to occupy the beam contour, which lead to phase errors for the nonfocal beams.

This idea later lead Zaghloul and Dong to propose a 360degree scanning lens in 2009 [40]. The proposed lens had symmetric beam and inner receiving contours, whose beam ports and receiving ports were interleaved. Beam port and receiving ports could maintain different modes of operations; it is possible to reuse the receiving port as beam port if circulators are adopted. Both nonfocal and 360-degree lenses were the topics in his dissertation.

In 2009 Uyguroğlu et al. [41] introduced a new concept of feed curves such that the phase error was reduced. The 
method was based on having three zero error positions on the radiating array for each feed curve point.

After 2009 various researchers are still trying to improve the design of the lens so as to achieve wide angle scanning with low lens loss and minimum phase error. Use of various existing optimization techniques, namely, genetic algorithm, particle swarm optimization, simulated annealing, and so forth, can come handy in improving the performance of the lens.

In view of all the above mentioned facts by various researchers it is quite clear that still there was a scope of improvement in the performance of the lens.

In 2012 Christie et al. [42] proposed a new type of broadband retrodirective array, which had been constructed using a microstrip Rotman lens. Automatic tracking of targets was obtained by exploiting the conjugate phase response of the beamforming network which was exhibited when the input ports were terminated with either open or short circuits. In addition, the true time-delay property of the Rotman lens gave broadband operation of the self-tracking array when used in conjunction with Vivaldi antennas.

In 2012 again Zhang et al. [43] described how a standard Rotman lens design could readily adapt in order to allow reconfigurable beam forming. This was achieved by applying concurrent excitations to the modified Rotman lens. A rationale for the design and underlying behavior of the modified, phase-aligned, Rotman lens as well as the deficiencies of a conventional Rotman lens in this mode of operation were provided.

In 2013 Zongxin et al. [44] developed a compact printable multibeam antenna array. The antenna system was composed of a printed Rotman lens and an antipodal dual elliptically tapered slot antenna array; both of the two components were studied, respectively, at first, and then integrated on a single printed circuit board to make up the integrated unit of the multibeam antenna array.

\section{Conclusion}

As the Rotman lens is a traveling wave structure it has to be sized in the order of several wavelengths to give a proper functionality. This leads to hard requirements considering the size of the Rotman lens, which could be determined by cost and size of the available substrate. Hence it was difficult to design Rotman lens at lower frequencies. Rotman lenses have proved to be a popular multiple beamforming technologies, due to their simplicity and performance. They have been overtaken by developments in digital signal processing and the speed of analogue to digital converters. The result is that many of these applications now use digital beamforming techniques. These applications basically operate at millimeter wavelengths, having bandwidths of many gigahertz or must drive so many beams.

The paper gives the insight details of the development in the area of the beamforming networks and Rotman lens antenna. Most of the work has been done to simplify the design equations related to beam port and array port of the lens to provide design freedom of the lens. The main aim was also to reduce the size of the lens by loading the parallel plates with the dielectric material. It was observed that if the lens was asymmetrical then the side lobe levels would increase. New design variable was introduced to reduce the phase error.

\section{Conflict of Interests}

The authors declare that there is no conflict of interests regarding the publication of this paper.

\section{References}

[1] J. Kim and F. Barnes, "Scaling and focusing of the Rotman lens," in Proceedings of the IEEE Antennas and Propagation Society International Symposium, vol. 2, pp. 773-776, July 2001.

[2] R. C. Hansen, Phased Array Antennas, John Wiley Sons, New York, NY, USA, 1998.

[3] S. S. D. Jones, H. Gent, and A. A. L. Browne, "Improvements in or relating to electromagnetic-wave lens and mirror systems," British Provisional Patent Specification No. 25926/56, 1956.

[4] K. Jaeheung, Developments of rotman lenses at micro/millimeterwave frequencies [Ph.D. thesis in Electrical Engineering], University of Colorado, Boulder, Colo, USA, 2003.

[5] M. Hawes and W. Liu, "Sparse array design for wideband beamforming with reduced complexity in tapped delay-lines," IEEE/ACM Transactions on Audio, Speech, and Language Processing, vol. 22, no. 8, pp. 1236-1237, 2014.

[6] D. H. Archer, "Fed multiple beam arrays," Microwave Journal, vol. 27, no. 9, pp. 13-195, 1984.

[7] H. Steyskal, C. Cox, and E. Ackerman, "Digital and optical beamforming techniques," in Phased Array Systems and Technology International Symposium, p. 616, 2003.

[8] C. W. Penney, R. J. Luebbers, and E. Lenzing, "Broad band Rotman Lens simulations in FDTD," in Proceedings of the IEEE Antennas and Propagation Society International Symposium and USNC/URSI Meeting, pp. 51-54, Washington, DC, USA, July 2005.

[9] G. L. Leonakis, "Correction to wide-angle microwave lens for line source applications," IEEE Transactions on Antennas and Propagation, vol. 34, no. 8, p. 1067, 1986.

[10] A. H. Jafari, W. Liu, and D. R. Morgan, "Study of sensor positions for broadband beamforming," IEEE Signal Processing Letters, vol. 20, no. 8, pp. 779-782, 2013.

[11] D. Archer, "Lens-fed multiple beam arrays," Microwave Journal, vol. 18, pp. 37-42, 1975.

[12] J. J. Lee and G. W. Valentine, "Multibeam array using Rotman lens and RF heterodyne," in Proceedings of the AP-S International Symposium on Antennas and Propagation Society, vol. 3, pp. 1612-1615, Baltimore, Md, USA, July 1996.

[13] A. Rahimian, "Microwave beamforming networks for intelligent transportation systems," in Intelligent Transportation Systems, A. Abdel-Rahim, Ed., pp. 123-142, InTech, Rijeka, Croatia, 2012.

[14] R. C. Hansen, "Design trades for Rotman lenses," IEEE Transactions on Antennas and Propagation, vol. 39, no. 4, pp. 464-472, 1991.

[15] W. Rotman and R. F. Turner, "Wide-angle microwave lens for line source applications," IEEE Transactions on Antenna and Propagation, vol. 11, no. 6, pp. 623-632, 1963. 
[16] J. Ruze, "Wide-angle metal-plate optics," Proceedings of the IRE, vol. 38, no. 1, pp. 53-59, 1950.

[17] D. H. Archer and M. J. Maybell, "Rotman lens development history at raytheon electronic warfare systems 1967-1995," in Proceedings of the IEEE Antennas and Propagation Society International Symposium, vol. 2, pp. 31-34, July 2005.

[18] D. H. Archer, R. J. Prickett, and C. P. Hartwig, "Multi-beam array antenna," U.S. Patent 3761936, 1973.

[19] A. Al-Zayed, L. Schulwitz, and A. Mortazawi, "A dual polarized millimeter-wave multibeam phased array," in Proceedings of the 2004 IEEE MITT-S International Microwave Symposium Digest, vol. 1, pp. 87-90, June 2004.

[20] J. Rao, "Multifocal three-dimensional bootlace lenses," IEEE Transactions on Antennas and Propagation, vol. 30, no. 6, pp. 1050-1056, 1982.

[21] J. Rao, "Correction to "Multifocal three-dimensional bootlace lenses',' IEEE Transactions on Antennas and Propagation, vol. 31, p. 541, 1983.

[22] J. P. Shelton, "Focusing characteristics of symmetrically configured bootlace lenses," IEEE Transactions on Antennas and Propagation, vol. 26, no. 4, pp. 513-518, 1978.

[23] M. J. Maybell, "Ray structure method for coupling coefficient analysis of the two dimensional Rotman lens," in Proceedings of the Antennas and Propagation Society International Symposium, pp. 144-147, Los Angeles, Calif, USA, June 1981.

[24] G. S. Hardie, R. Hernandez, and M. J. Maybell, "Parallel plate lens antenna," U.S. Patent 4490723, 1984.

[25] T. Katagi, S. Mano, and S. Sato, "An improved design method of rotman lens antennas," IEEE Transactions on Antennas and Propagation, vol. 32, no. 5, pp. 524-527, 1984.

[26] L. Musa and M. Smith, "Microstrip rotman lens port design," in Proceedings of the Antennas and Propagation Society International Symposium, vol. 24, pp. 899-902, 1986.

[27] L. Musa and M. S. Smith, "Microstrip port design and sidewall absorption for printed Rotman lenses," IEE Proceedings $H$ : Microwaves, Antennas and Propagation, vol. 136, no. 1, pp. 53$58,1989$.

[28] L. Hall, H. Hansen, and D. Abbott, "Rotman lens for mmwavelengths," in Smart Structures, Devices, and Systems, vol. 4935 of Proccedings of SPIE, pp. 215-221, 2002.

[29] D. R. Gagnon, "Procedure for correct refocusing of the Rotman lens according to Snell's law," IEEE Transactions on Antennas and Propagation, vol. 37, no. 3, pp. 390-392, 1989.

[30] P. K. Singhal, R. D. Gupta, and P. C. Sharma, "Design and analysis of modified Rotman type multiple beam forming lens," in Proceedings of the IEEE Region 10 International Conference on 'Global Connectivity in Energy, Computer, Communication and Control (TENCON '98), vol. 2, pp. 257-260, December 1998.

[31] M. J. Maybell, "Printed Rotman Lens fed array having wide bandwidth low side lobes, constant beam width and synthesized radiation pattern," in Proceedings of the IEEE AP Symposium Digest, pp. 373-376, Houston, Tex, USA, 1983.

[32] E. O. Rausch and A. F. Peterson, "Rotman lens design issues," in Proceedings of the IEEE Antennas and Propagation Society International Symposium and USNC/URSI Meeting, vol. 2, pp. 35-38, July 2005.

[33] P. K. Singhal, P. C. Sharma, and R. D. Gupta, "Rotman lens with equal height of array and feed contours," IEEE Transactions on Antennas and Propagation, vol. 51, no. 8, pp. 2048-2056, 2003.

[34] P. Simon, "Analysis and synthesis of Rotman lenses," in Proceedings of the 22nd AIAA International Communications Satellite Systems Conference \& Exhibit, Monterey, Calif, USA, May 2004.
[35] J. Dong and R. Cheung, "A computer synthesized 2 8 GHz printed rotman Lens with 98 input-to-output configuration," in Proceedings of the 2011 IEEE International Symposium on Antennas and Propagation and USNC/URSI National Radio Science Meeting (APSURSI '11), pp. 616-618, July 2011.

[36] J. Dong, A. I. Zaghloul, R. Sun, and C. J. Reddy, "EHF rotman lens for electronic scanning antennas," in Proceedings of the Asia Pacific Microwave Conference (APMC '08), December 2008.

[37] J. Dong, A. I. Zaghloul, and R. Rotman, "Non-focal minimumphase-error planar rotman lens," in Proceedings of theUSNC /URSI National Radio Science Meeting, Boulder, Colo, USA, January 2008.

[38] J. Dong, A. I. Zaghloul, and R. Rotman, "Non-focal minimumphase-error planar rotman lens," in Proceedings of the URSI National Radio Science Meeting, Boulder, Colo, USA, 2008.

[39] J. Dong and A. I. Zaghloul, "Implementation of microwave lens for 360-degree scanning," in Proceeding of the IEEE International Symposium on Antennas and Propagation (APSURSI '09), pp. 1-4, Charleston, SC, USA, June 2009.

[40] A. I. Zaghloul and J. Dong, "A concept for a lens configuration for $360^{\circ}$ scanning," IEEE Antennas and Wireless Propagation Letters, vol. 8, pp. 985-988, 2009.

[41] R. Uyguroğlu, A. Y. Öztoprak, and C. Ergün, "Improved phase performance for rotman lens," International Journal of RF and Microwave Computer-Aided Engineering, vol. 23, no. 6, pp. 634638, 2012.

[42] S. Christie, R. Cahill, N. B. Buchanan et al., "Rotman lensbased retrodirective array," IEEE Transactions on Antennas and Propagation, vol. 60, no. 3, pp. 1343-1351, 2012.

[43] Y. Zhang, S. Christie, V. Fusco, R. Cahill, G. Goussetis, and D. Linton, "Reconfigurable beam forming using phase-aligned Rotman lens," IET Microwaves, Antennas and Propagation, vol. 6, no. 3, pp. 326-330, 2012.

[44] W. Zongxin, X. Bo, and Y. Fei, "A multibeam antenna array based on printed rotman lens," International Journal of Antennas and Propagation, vol. 2013, Article ID 179327, 6 pages, 2013. 

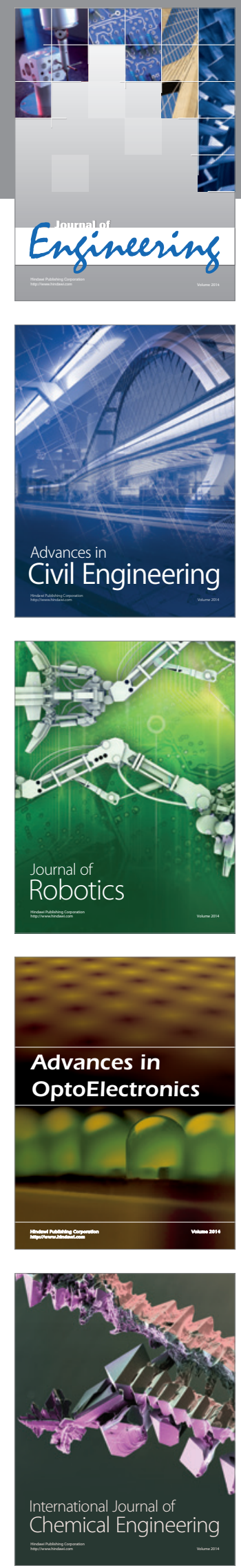

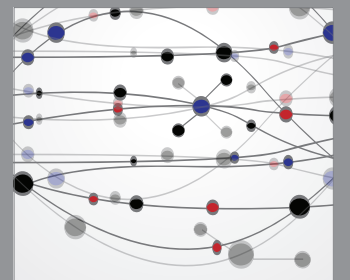

The Scientific World Journal
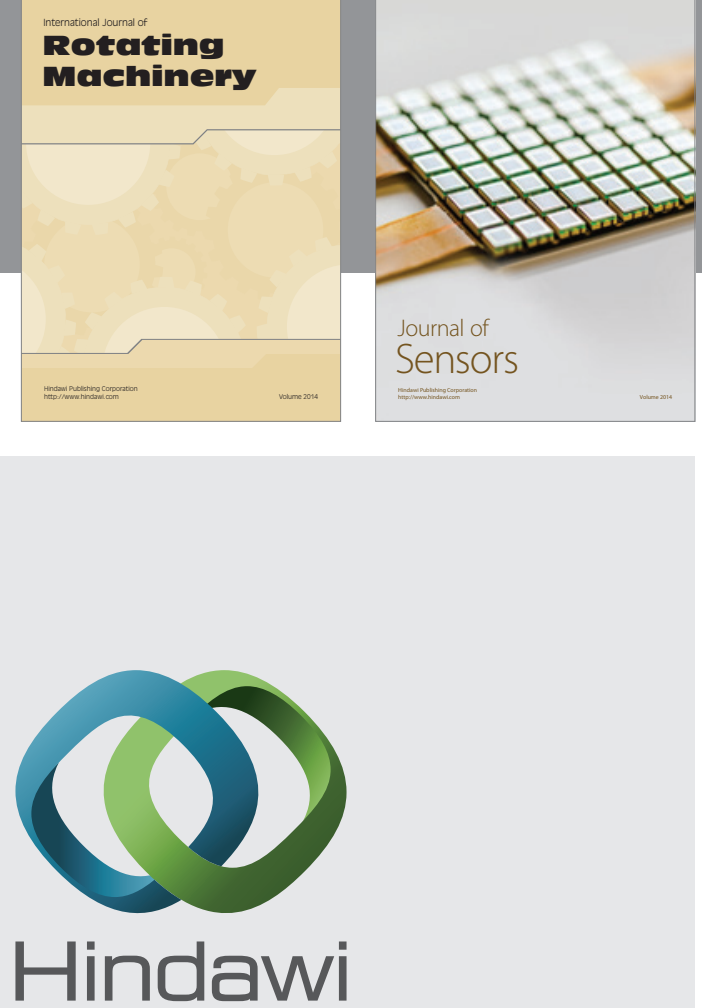

Submit your manuscripts at http://www.hindawi.com
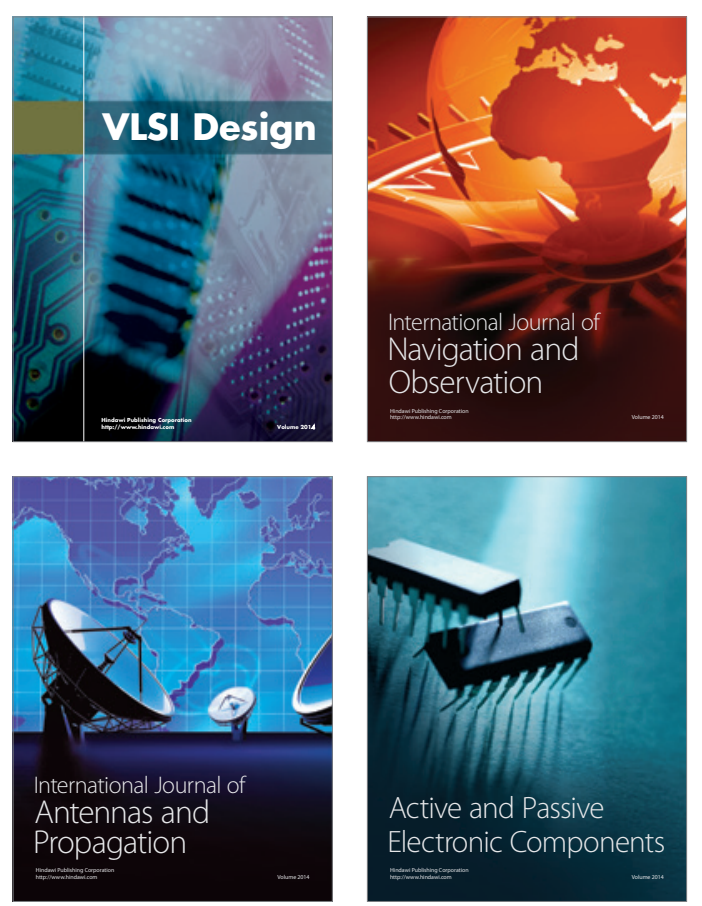
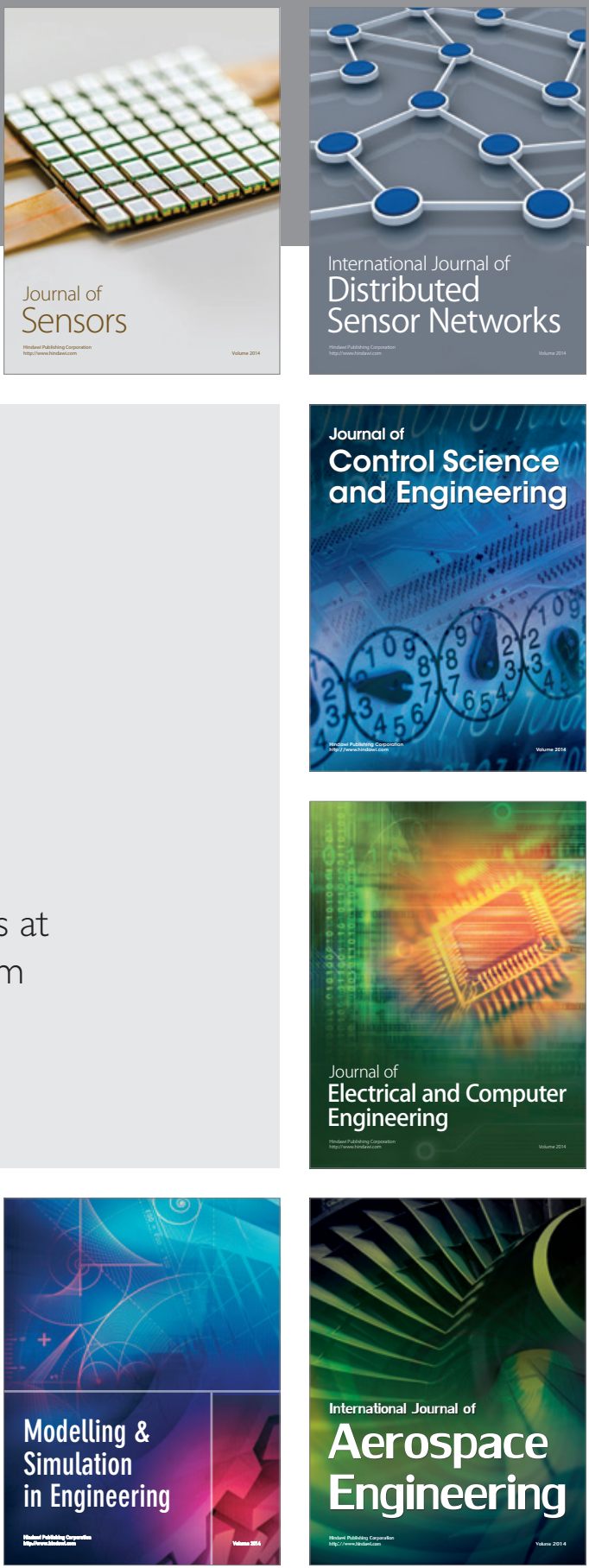

Journal of

Control Science

and Engineering
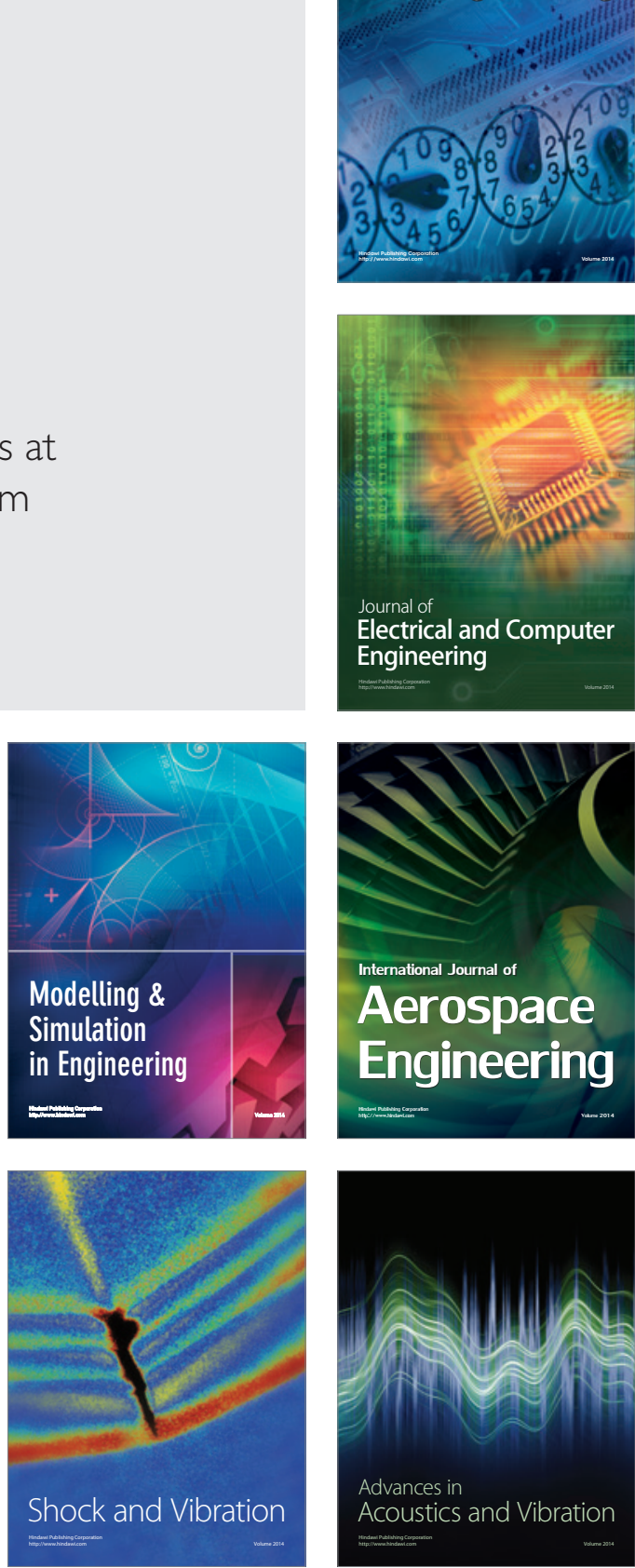